\title{
Comparative analysis of selected classifiers in posterior cruciate ligaments computer aided diagnosis
}

\author{
P. ZARYCHTA*, P. BADURA, and E. PIETKA \\ Silesian University of Technology, Faculty of Biomedical Engineering, 40 Roosevelta St., 41-800 Zabrze, Poland
}

\begin{abstract}
A study on computer aided diagnosis of posterior cruciate ligaments is presented in this paper. The diagnosis relies on T1-weighted magnetic resonance imaging. During the image analysis stage, the ligament region is automatically detected, localized, and extracted using fuzzy segmentation methods. Eight geometric features are defined for the ligament object. With a clinical reference database containing 107 cases of both healthy and pathological cases, a Fisher linear discriminant is used to select 4 most distinctive features. At the classification stage we employ five different soft computing classifiers to evaluate the feature vector suitability for the computerized ligament diagnosis. Among the classifiers we introduce and specify the particle swarm optimization based Sugeno-type fuzzy inference system and compare its performance to other established classification systems. The classification accuracy metrics: sensitivity, specificity, and Dice index all exceed $90 \%$ for each classifier under consideration, indicating high level of the proposed feature vector relevance in the computer aided ligaments diagnosis.
\end{abstract}

Key words: posterior cruciate ligament, computer aided diagnosis, feature extraction, classification, soft computing.

\section{Introduction}

The primary function of the joints in the human body is to ensure the smooth movement of the limbs. The knee joint is the largest and most complex of these joints. The anatomy of the knee joint is the reason for which it is considered in the medical theory as very sensitive, fragile and frequently susceptible to injuries [1]. Anterior cruciate ligaments (ACL) and posterior cruciate ligaments (PCL) are elements of this joint. These ligaments are particularly vulnerable to injuries. Cruciate ligaments connect very strongly the femur and the tibia. ACLs and PCLs are also a vital stabilizer of the knee. The main functions of both cruciate ligaments are as follows: they ensure proper kinematics of the knee joint, take care of the smooth movement of the knee joint and protect the articular cartilage [2,3]. These ligaments (especially PCL) also stabilize the knee against excessive varus or valgus angulation. Cruciate ligaments of the knee joint always remain partially strained as a result of the non-uniform shape and unequal length of their fibers. For this reason these ligaments are vulnerable to injuries, especially in the case of abrupt and unnatural movements of the knee [4, 5].

Nowadays, injuries of cruciate ligaments are a plague for both athletes and nonathletes (especially for active people and the elderly). ACL and PCL injuries constitute approximately $60 \%$ and $25 \%$ of knee injuries, respectively [6]. Generally injuries of the cruciate ligaments are a common cause of chronic knee instability and usually limit even ordinary daily activities, such as walking on uneven surfaces. A problem with the ACL or PCL formerly meant the end of an active life. Nowadays it has changed, but it is still particularly important to make a fast and correct diagnosis.

*e-mail: piotr.zarychta@polsl.pl
Usually, in case of PCLs injuries, the fibers of the ligament tear apart over its entire length, which results in ligament swelling. Therefore, the swelling of the ligament is a fundamental criterion of the PCL rupture. Among the PCL injuries complete tears (occur in approximately $40 \%$ of cases), partial tears $(50 \%)$ and avulsion tears $(7 \%)$ are distinguished [6-8]. In this case the diagnostic method of choice is the magnetic resonance imaging (MRI, the elementary method for diagnostics of the knee joint, especially the cruciate ligaments injuries). MRI allows the visualization of PCLs throughout their length and gives the possibility to assess the shape and internal structure of cruciate ligaments in a completely non-invasive manner. MRI of the knee joint is typically performed on T1-weighted sequences and different varieties of T2-weighted sequences, usually in three planes: transverse, coronal and sagittal. The pathological PCL has a striated appearance whereas the normal PCL has a uniform low intensity signal. It has a slightly arcuate shape and smoothly goes from attachment to the femur to attachment to the tibia. PCL is well visible in the sagittal plane. Usually this structure appears on 4-6 slices of the T1- or T2-weighted MRI sequences of the knee joint [9]. For that reason, in this paper the computerized analysis of PCLs is applied to MRI of the knee joint.

A review of the literature shows that there is a lack of works describing the methods of computer assisted diagnosis of cruciate ligaments. Many publications are focused on the two most important problems: the process of reconstruction [7, 8, 10-13], and then the rehabilitation [8, 14-16] of the ACL and PCL, respectively. This paper is a continuation of work presented in [1719] and fills in this gap providing in the first step a description of feature extraction of both, normal and pathological structures of the posterior cruciate ligament and then a comparative analysis of selected classifiers in the computerized diagnostics of those ligaments. The article describes how, on the basis of MRI images of the knee joint, the radiologist or orthopedist may, in a non-in- 


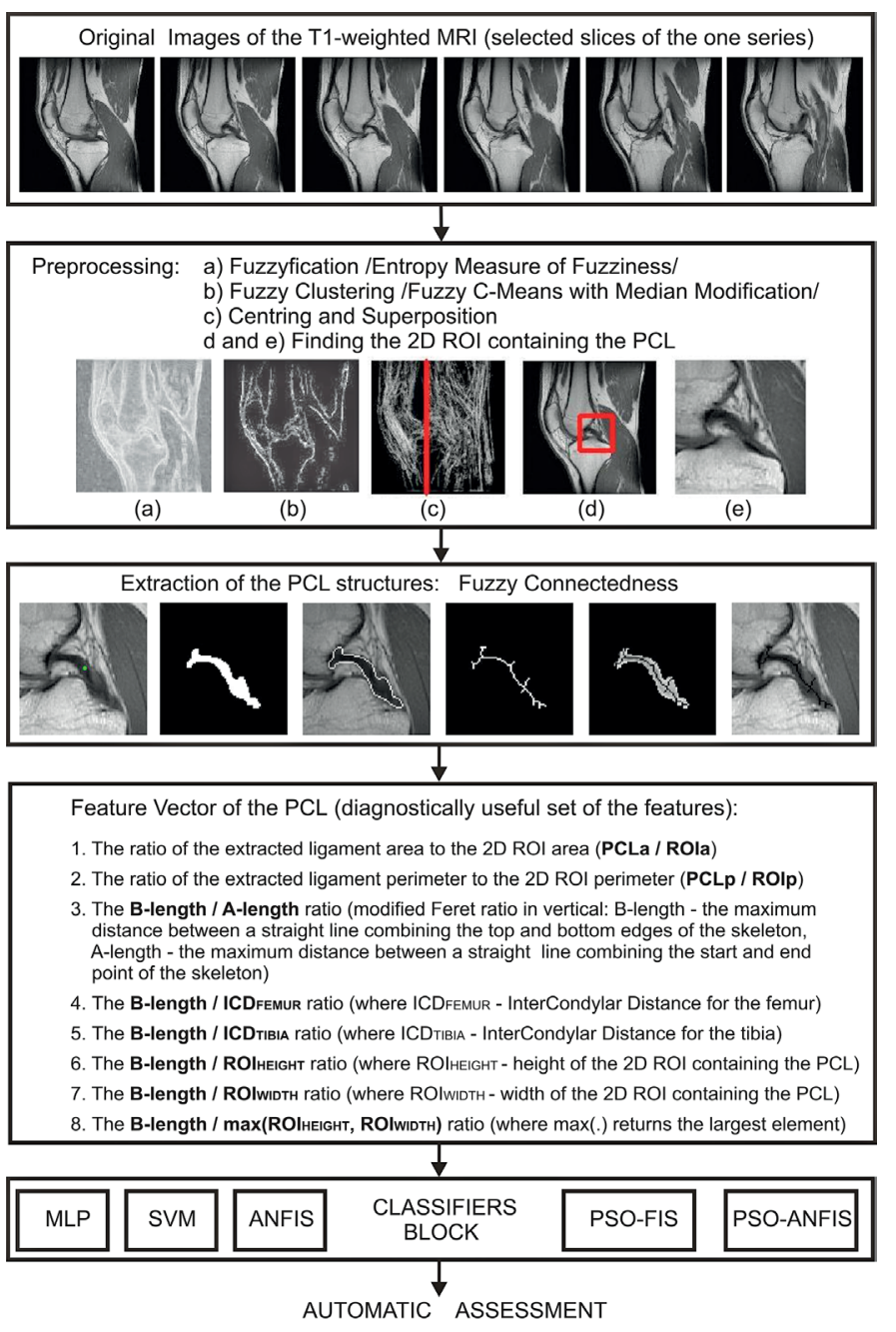

Fig. 1. Block diagram of the study

vasive way, obtain some important information relevant to the diagnosis of PCL. From the clinical point of view, the proposed method can be particularly helpful in cases of doubt relating to PCL pathology (partial tears), where the visual assessment may be questionable. This information, may significantly improve and facilitate the process of diagnosis of those ligaments.

The paper introduces several novelties. First, the paper is presenting the feature extraction process of the PCLs. It intro- duces new elements to the feature vector describing the PCLs. Second, the paper describes the discriminant analysis and feature space reduction followed by comparative analysis of five selected classifiers in the computer diagnostics of the ligaments. It indicates the most important diagnostic features of the ligament. Among the soft computing classifiers we introduce and specify the novel particle swarm optimization based fuzzy inference system and compare its performance with some well known and established classification tools. The evaluation stage relies on a clinical reference database containing 107 knee joint MRI cases each with expert assessment on potential cruciate ligament pathology.

The PCL feature extraction process contains several phases (Fig. 1). First, the preprocessing of medical images (T1-weighted MRI of the knee joint in the sagittal plane) is performed. It includes stages required to obtain a two-dimensional region of interest (2D ROI), since the extraction of 2D ROI can significantly reduce the computational complexity. Then, the extraction phase is implemented. Finally, the feature vector of the PCL is created and validated. In this paper the procedure of finding a 2D ROI and the procedure of PCL extraction are not described in detail. A detailed description thereof can be found in [20,21].

The paper is organized as follows. Section 2 describes the PCL feature extraction process. Five Classifiers employed for the computer aided PCL diagnosis are introduced and described in Section 3. The clinical database used for evaluation is presented in Section 4 along with the results obtained at both, the feature extraction, and classification stages. The methodology and results are discussed in Section 4. Section 5 concludes the paper.

\section{Feature extraction}

A properly selected set of features is key to the development of a computer assisted diagnosis system. Such set can greatly facilitate the diagnosis of selected anatomical structures. In other words the main purpose of extracting a feature vector describing the PCL structures is to classify these structures into normal or pathological ones. In this paper, only a diagnostically sensitive set of features, that differentiates one class from the others, has been described. These features belong to two main groups: features based on the 2D ROI (Figs. 2a, b, e) containing the extracted ligament structures: $\mathbf{P C} \mathbf{L}_{a} / \mathbf{R O I}{ }_{a}$ ratio (the ratio of the extracted ligament area to the 2D ROI area), a)

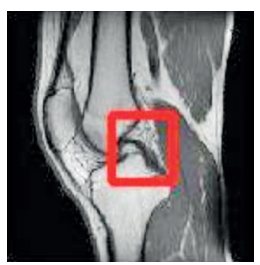

b)

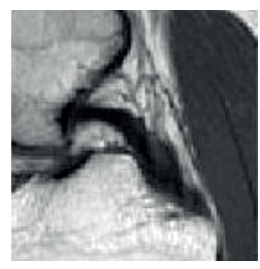

c)

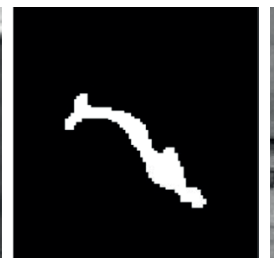

d)

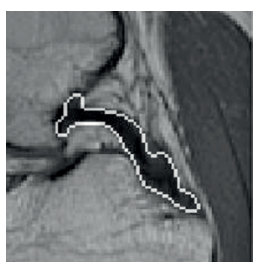

e)

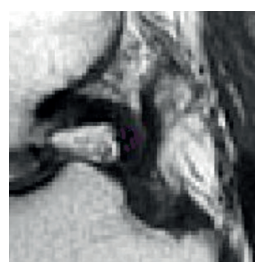

f)

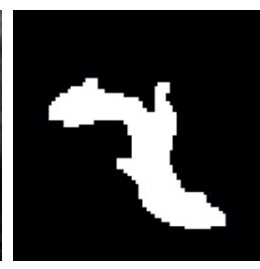

g)

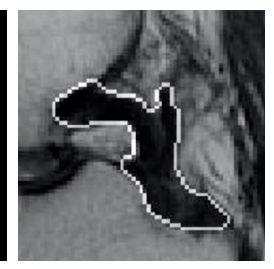

Fig. 2. Features based on the 2D ROI containing the extracted PCL structure, a) selected slice from the T1-weighted MRI with marked 2D ROI, b) 2D ROI containing the normal PCL, c) area of the normal extracted ligament $\left(\mathbf{P C} \mathbf{L}_{a}\right)$, d) perimeter of the normal extracted ligament $\left.\left(\mathbf{P C} \mathbf{L}_{p}\right\}\right)$, e) 2D ROI containing the pathological PCL, f) area of the pathological extracted ligament $\left(\mathbf{P C} \mathbf{L}_{a}\right)$, g) perimeter of the pathological extracted ligament $\left(\mathbf{P C L}_{p}\right)$ 
a)

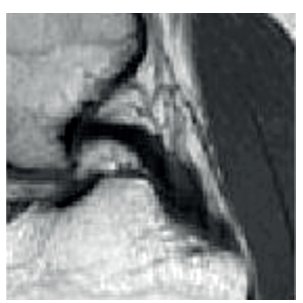

b)

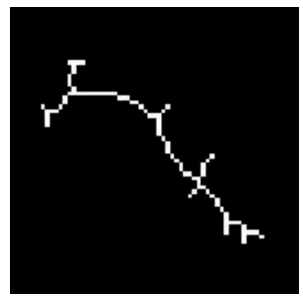

c)

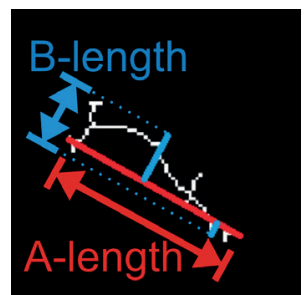

d)

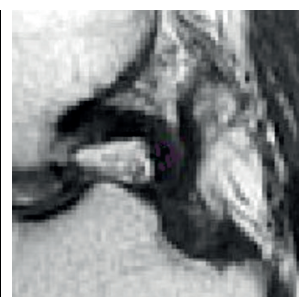

e)

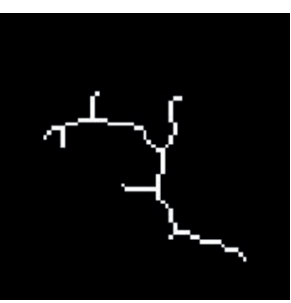

f)

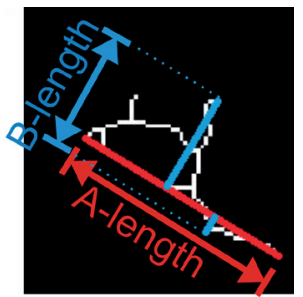

Fig. 3. Features based on the skeleton of the extracted PCL structure, a) 2D ROI containing the normal PCL, b) skeleton of the normal extracted ligament, c) skeleton with marked A- and B-length for normal PCL, d) 2D ROI containing the pathological PCL, e) skeleton of the pathological extracted ligament, f) skeleton with marked A- and B-length for pathological PCL

$\mathbf{P C L}_{p} / \mathbf{R O I}_{p}$ ratio (the ratio of the extracted ligament perimeter to the 2D ROI perimeter) and features based on the skeleton of the extracted PCL: B/A ratio (B-length/A-length - the modified Feret ratio in vertical, where B-length denotes the maximum distance between a straight line linking the top and bottom edges of the skeleton and A-length denotes the maximum distance between a straight line connecting the start and end point of the skeleton), B/ICD $\mathbf{I}_{F E M U R}$ (where the $\mathbf{I C D}_{F E M U R}$ denotes the intercondylar distance for the femur), $\mathbf{B} / \mathbf{I C D}_{\text {TIBIA }}$ (where the ICD $\mathbf{D}_{\text {TIBIA }}$ denotes the intercondylar distance for the tibia), B/ROI ${ }_{H E I G H T}$ (where the ROI $\mathbf{R E I G H T}_{\text {denotes the height }}$ of the 2D ROI containing the PCL), B/ROI $\mathbf{R O I}_{W I D T H}$ denotes the width of the 2D ROI containing the $\mathrm{PCL}), \mathbf{B} / \mathbf{m a x}\left(\mathbf{R O I} \mathbf{I}_{H E I G H T}, \mathbf{R O I}_{\text {WIDTH}}\right.$ ) (where $\max ($.$) returns$ the largest element).

The surface area of the extracted structure of the ligament (Figs. 2c, f) seems to be the most natural feature. However, this feature is diagnostically useful only when being independent of the size of the patient's knee joint. Otherwise, it may lead to misclassification of the extracted ligament (a large area of a normal PCL in case of a large knee joint size can be misdiagnosed as pathological structure). Therefore, it is necessary to relate the area of the extracted PCL structure to the area of the 2D ROI containing this structure. The 2D ROI depends on the anatomy of the knee joint. Thus, the feature that contains information about the area of the ligament, and takes into account the size of the patient is the $\mathbf{P C} \mathbf{L}_{a} / \mathbf{R O I}{ }_{a}$ ratio. In order to calculate this ratio, it is necessary to determine the area of the ligament $\left(\mathbf{P C} \mathbf{L}_{a}\right)$. Depending on the slice thickness, the PCL structure may be visible in 4-6 slices in the sagittal plane. All these slices are subjected to the area extraction procedure. For each patient the slice containing the largest area of the extracted ligament has been selected.

A very similar procedure has been used to calculate the second feature based on the $2 \mathrm{D}$ ROI - the $\mathbf{P C} \mathbf{L}_{p} / \mathbf{R O I} p$ ratio, where $\mathbf{P C L} \mathbf{L}_{p}$ (Figs. 2d, g) denotes the perimeter of the extracted ligament (for the slice containing the largest area of the extracted PCL), and $\mathbf{R O I}_{p}$ denotes the perimeter of the 2D ROI. Also in this case it is necessary to relate the perimeter of the extracted PCL structure to the perimeter of the 2D ROI in order to make the feature independent of the size of the knee joint.

As mentioned above, the second group of features is based on the skeleton of the extracted ligament (Figs. 3b, e). On the basis of this skeleton, two elementary lengths may be determined: A-length and B-length, respectively. A-length denotes the maximum distance between a straight line connecting the start and end point of the skeleton (Figs. 3c, f). B-length denotes the maximum distance (perpendicular to A-length) between the top and bottom edge of the skeleton (Figs. 3c, f). In practical applications it is important to calculate the $\mathbf{B} / \mathbf{A}$ ratio. This ratio features a high discriminant power, especially in the diagnostics of PCL tissue.

Next two features make use of the information about the intercondylar distance for both the head of the femur (ICDFEMUR $)$ and the head of the tibia (ICD TIBIA $_{\text {, }}$, respectively. The intercondylar distance is the distance between the left and right condyle of the femoral or tibial head (Fig. 4). On the basis of the information about the B-length and both intercondylar distances ICD $_{F E M U R}$ and ICD $\mathbf{I I B I A}_{\text {, two diagnostically useful ratios can }}$ be calculated: B/ICD $\mathbf{D}_{F E M U R}$ and $\mathbf{B} / \mathbf{I C D}_{\text {TIBIA }}$ respectively.

Both $\mathbf{B} / \mathbf{I C D} \mathbf{D}_{\text {TIBIA }}$ and $\mathbf{B} / \mathbf{I C D} \mathbf{D}_{\text {FEMUR }}$ ratios are well suited for practical tasks. Nevertheless these two ratios have a basic disadvantage, the entire T1-weighted MRI knee joint slice $(256 \times 256$ pixels $)$ has to be subjected to the analysis. Therefore, both these expressions are modified to $\mathbf{B} / \mathbf{R O I} \mathbf{I}_{H E I G H T}$ and $\mathbf{B} / \mathbf{R O I}{ }_{W I D T H}$, where $\mathbf{R O I}_{H E I G H T}$ and $\mathbf{R O I} \mathbf{I}_{W I D T H}$ denote the 2D ROI height and width, respectively. In this case, the analysed region is reduced by over 6 times $(100 \times 100$ pixels $)$. Preliminary studies indicate comparable results.

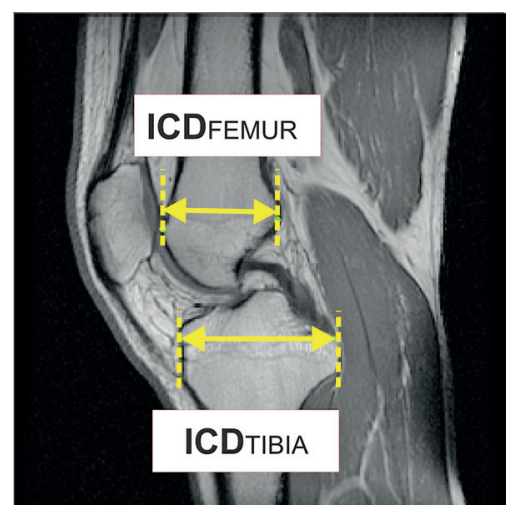

Fig. 4. Selected slice from the T1-weighted MRI with marked intercondylar distances $\mathrm{ICD}_{\text {FEMUR }}$ and $\mathrm{ICD}_{\text {TIBIA }}$ 


\section{Classification}

Five different soft computing classifiers have been used at the decision making process: the artificial neural network multilayer perceptron (MLP), the support vector machine (SVM), the adaptive neuro-fuzzy inference system (ANFIS), the particle swarm optimized fuzzy inference system (PSO-FIS), and the particle swarm optimized ANFIS (PSO-ANFIS). Each one is supplied with features selected from the entire set described in Section 2. The feature selection process involves Fisher linear discriminant (FLD). Structures and specifications of classifiers have been - where necessary - established experimentally with various settings involved. The efficiency metrics were analysed as main factors with another identified in architectural simplicity and low operating time.

3.1. Feature selection. Fisher linear discriminant. The feature vector has been subjected to the Fisher linear discriminant analysis [23] at a confidence level $p=0.05$. The reference set for FLD analysis is constructed using the entire clinical database available at the experimental stage and described in Section 4. The FLD analysis has reduced the number of features to 4 , yielding the following vector:

$$
\left[\begin{array}{l}
\mathbf{P C L} \\
\text { B/ROI } \\
\mathbf{B} / \mathbf{A} \\
\mathbf{B} / \mathbf{I C D} \\
\mathbf{B} / \mathbf{m a x}\left(\mathbf{R O I}_{H E I G H T}, \mathbf{R O I}_{W I D T H}\right)
\end{array}\right] .
$$

Thus, each classifier under consideration has four inputs supplied with selected features and a single output producing a binary decision.

3.2. Multilayer perceptron. The MLP classifier [24] consists of 2 hidden layers with 5 and 3 units, respectively. All hidden neurons have hyperbolic tangent activation function. The output activation function has a linear form $\phi(x)=x$ cut off at 0 and 1.
The real-valued output $y_{M L P}$ is binarized by thresholding at 0.5 to produce the decision $Y_{M L P}$ :

$$
Y_{M L P}=\left\{\begin{aligned}
1 & \Leftrightarrow y_{M L P} \geq 0 \\
0 & \Leftrightarrow y_{M L P}<0 .
\end{aligned}\right.
$$

3.3. Support vector machine. The SVM classifier is used as a second classification tool $[25,26]$. SVM divides the problem space into two subspaces by determining a hyperplane separating the training samples. Here we use a canonic two-class SVM.

\subsection{Adaptive neuro-fuzzy inference system. The ANFIS} merges artificial neural networks and fuzzy inference systems for decision making purposes including clustering and classification [27]. Here, we employ a Sugeno-type [28] fuzzy inference system (FIS) with Gaussian input membership functions and linear output membership functions, initiated using fuzzy a c-means data clustering [29]. The number of membership functions per FIS input/output is set to 4, since it consistently provides the best efficiency metrics in a range of $[2,5]$.

3.5. Particle swarm based fuzzy inference system. The PSO is a swarm intelligence technique for optimization problems [30]. It involves basic agent and swarm behavior principles [31] in order to perform intelligent survey through a multidimensional problem space. PSO is willingly employed for classification, pattern recognition, and machine learning approaches also in the medical image analysis domain [32-36].

In this paper we propose a fuzzy inference system (FIS) [37] trained in terms of a PSO. The Sugeno-type FIS [28] uses three Gaussian membership functions per each input and three linear output membership functions. The knowledge base consists of $3^{4}=81$ fuzzy rules with unique combinations of four inputs' linguistic values (antecedent). Each PSO particle is characterized by its location $\mathbf{x}=\left\{x_{i}: i=1 \ldots N\right\}$, where $N$ denotes the feature space dimensionality. Each feature corresponds to a single FIS parameter in terms of input and output membership functions, as well as fuzzy rules expressions (Fig. 5). A par-

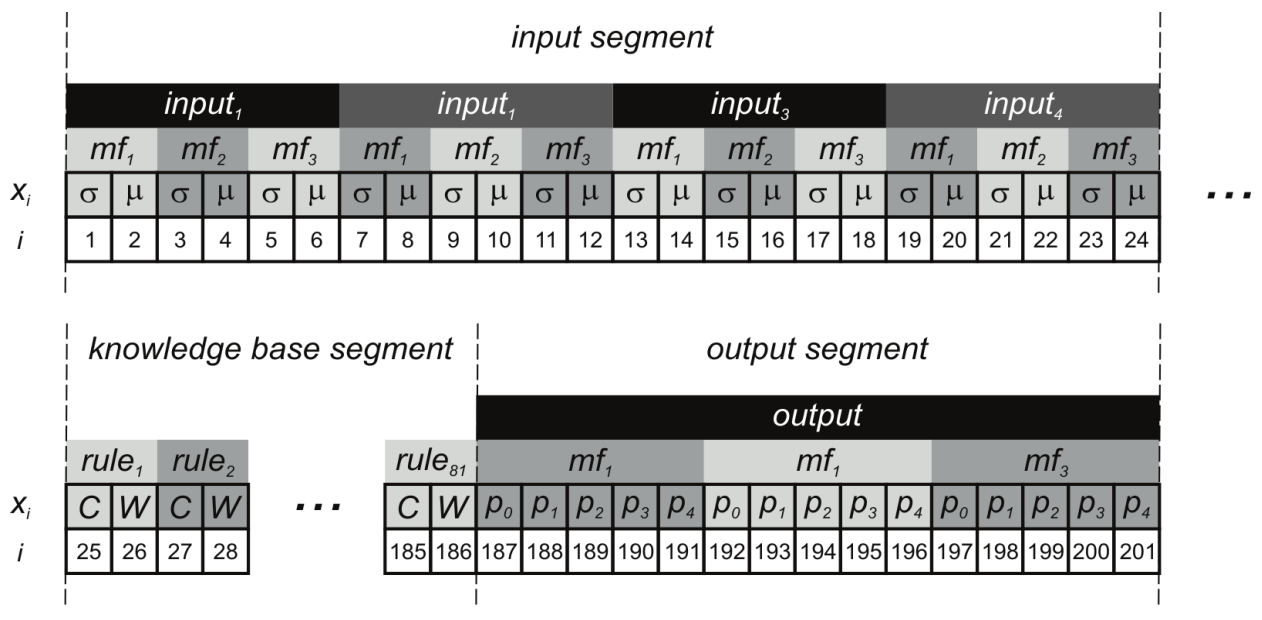

Fig. 5. Specification of the PSO-FIS particle. Input segment denotations: input $t_{j}-j$-th FIS input, $m f_{k}-$ Gaussian membership function for $k$-th fuzzy value with parameters $\mu, \sigma$. Knowledge base segment denotations: rule $j$-j-th fuzzy rule with weight $W$ and consequent $C$. Output segment denotations: $m f_{j}$ - membership function for $k$-th output fuzzy value with parameters $p_{k}: k=0 \ldots 4$ 
ticle from Fig. 5 operates in a 201-dimensional feature space, though we have also tested different FIS settings with automatic space dimensionality adjustment. Note, that there is a set of constraints limiting some coordinates (i.e. $\sigma>0$ for every input Gaussian function; in the knowledge base segment each rule consequent $C \in\{1,2,3\}$ and each rule weight $W \in[0,1]$ ).

A particle $\mathbf{x}$ is evaluated in terms of a fitness function $f i t(\mathbf{x})$ as a mean squared error (MSE) over a training set $T$ subjected to fuzzy inference using $\mathbf{x}$-related FIS:

$$
f i t(\mathbf{x})=\operatorname{MSE}(\mathbf{x}, T)=\frac{1}{\|T\|} \sum_{t \in T}\left(y-y_{t}\right)^{2},
$$

where $y$ denotes the FIS output value, whilst $y_{t} \in\{0,1\}$ is the expert assessment of the $t$-th case.

The following agent and swarm parameters and mechanisms have been established during the classifier testing:

- the swarm consists of 50 particles moving around in 500 iterations, unless the best fitness reaches 0.01 or it did not improve throughout the last 250 iterations.

- Particles are initiated pseudorandomly with all individual coordinates' constraints secured.

- The particle velocity calculation is affected by three factors: (a) inertia with intensity decreasing in time, (b) particle's best location memory, (c) particle neighborhood's best location memory [30].

- The particle maximum velocity is limited in terms of each coordinate in order to secure the swarm stability.

3.6. Particle swarm based artificial neuro-fuzzy inference system. Two latter classifiers were combined in order to introduce the fifth tool: the PSO-ANFIS [38]. Namely, when the ANFIS training is finished (Section 3.4), it is passed in several copies ( $10 \%$ of the swarm population) to the PSO-FIS approach. The remainder of the swarm is initiated pseudorandomly as described in Section 3.5. All agent and swarm parameters are set as in the PSO-FIS case. During the PSO training the original ANFIS is subjected to changes, also in terms of the number and formulae of fuzzy rules.

3.7. Classifier training and testing procedure. Each classifier training and testing procedure involves a $k$-fold cross validation scheme with various $k$. The reference database is each time divided into $k$ groups and then in each of the $k$ experiments a single group is used as a testing set, whilst the remainder constitute a training set [39]. The classification efficiency is evaluated using sensitivity, specificity, and Dice index measures:

$$
\begin{gathered}
\text { Sen }=\frac{T P}{T P+F N} \cdot 100 \%, \\
\text { Spec }=\frac{T N}{T N+F P} \cdot 100 \%, \\
D=\frac{2 \cdot T P}{2 \cdot T P+F N+F P} \cdot 100 \%,
\end{gathered}
$$

where $T P, F N, T N, F P$ denote the number of true positive, false negative, true negative and false positive PCL pathology detections, respectively.

\section{Results and discussion}

4.1. Materials. The study has been tested on 107 clinical T1-weighted MRI studies of the knee joint. The MRI data (17-24 slices per volume) have been acquired in the sagittal plane for females and males at different age. This clinical group consisted of 62 normal and 45 pathological cases of PCLs. The pathological cases included the following injuries: ligament rupture of the "shaving brush" type, partial tear, extensive partial tear and ligament displacement as a result of femur head fracture (including complete PCL's interruption) [40].

4.2. Feature extraction results. The analysis of the study results was based on the assessment performed by three independent experts: two radiologists and one orthopedist. These experts represent three medical centres (hospitals). Fig. 6 shows a)
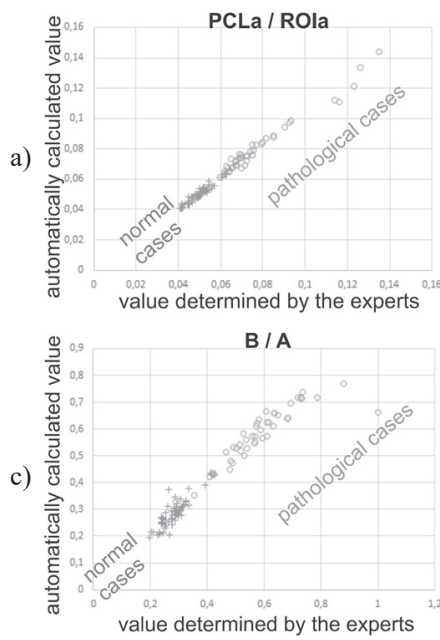
B / ICDTIBIA

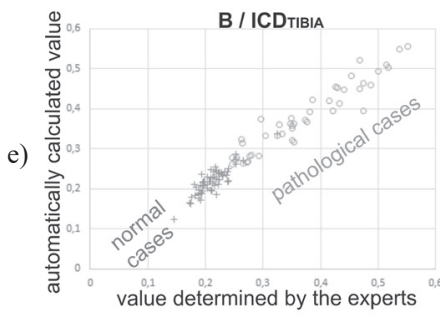

B / ROlwIDTH

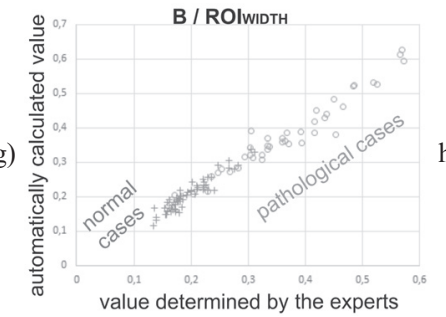

b)

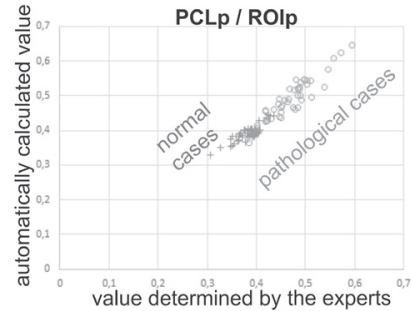

B / ICDFEMur

d)

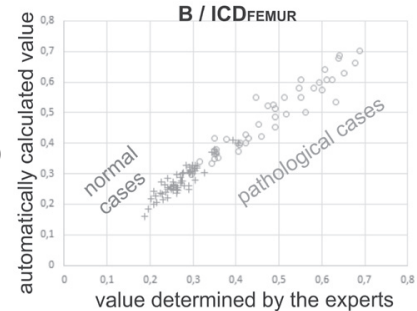
B / ROIHEIGHT

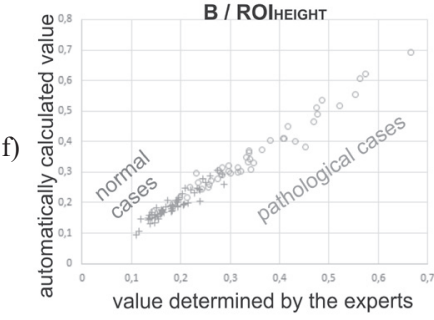

B / max(ROIHEIGHT, ROIWIDTH)

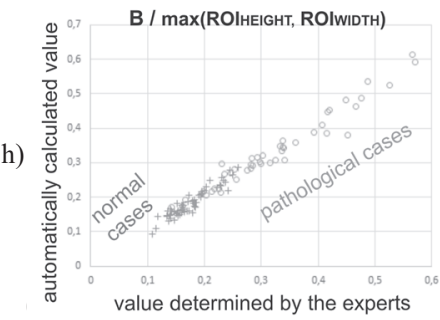

Fig. 6. Scatterplots of the following ratios: a) $\mathbf{P C L} \mathbf{L}_{a} / \mathbf{R O I}_{a}$ b) $\mathbf{P C L} \mathbf{L}_{p} / \mathbf{R O I} \mathbf{I}_{p}$

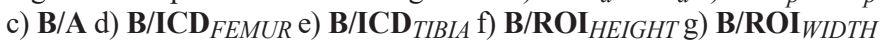
h) $\mathbf{B} / \mathbf{m a x}\left(\mathbf{R O I}_{H E I G H T}, \mathbf{R O I}_{\text {WIDTH }}\right)$ 
a comparison, in the form of scatterplots, of the values of elements of the feature vector: $\mathbf{P C L}_{a} / \mathbf{R O I} \mathbf{I}_{a}, \mathbf{P C L}_{p} / \mathbf{R O I} \mathbf{I}_{p}, \mathbf{B} / \mathbf{A}$, $\mathbf{B} / \mathbf{I C D}_{F E M U R}, \mathbf{B} / \mathbf{I C D}_{T I B I A}, \mathbf{B} / \mathbf{R O I} \mathbf{I}_{H E I G H T}, \mathbf{B} / \mathbf{R O I} \mathbf{I}_{W I D T H}$, $\mathbf{B} / \mathbf{m a x}\left(\mathbf{R O I}_{H E I G H T}, \mathbf{R O I}_{W I D T H}\right)$. In Fig. 6 the X-axis represents the values determined by experts (average value of three marks given by the experts) while the Y-axis specifies the automatically calculated values of the individual ratios.

Based on each of these ratios, two cases can be distinguished: normal and pathological, respectively. The analysis of scatterplots shown in Figs. 6 indicates that for the normal PCLs the results are more concentrated around the mean (the variances calculated for each of ratios of the segmented structures are lower). Whereas, for the pathological cases of PCLs the scattering of the results is large (variances are several times higher than for the normal cases).

4.3. Classification results. Each of the five classifiers described in Section 3 was subjected to a $k$-fold cross validation procedure with three different $k: 3,10$ and 107. The latter choice corresponds to a leave-one-out cross validation as a special case of a $k$-fold scheme. In order to increase the evaluation reliability, each $k$-fold cross validation experiment was repeated $N=20$ times, each with its own independent dataset organized into $k$ groups. The classification efficiency summary is gathered in Table 1. The average numbers of TP, FP, TN, FN and combined sensitivity and specificity values are presented in the table, as well as the best case metrics throughout $N$ runs in terms of a Dice index.
The mean classification sensitivity ranges between 94.8 and $98.4 \%$, specificity between 90.1 and $93.3 \%$, and Dice index between 94.1 and $96.8 \%$ throughout all classifiers and cross validations. The most accurate, consistent, and repeatable results are yielded by the SVM classifier followed by the ANFIS, which indicates their relatively low sensitivity to initiation. On the other hand, the MLP system offers the lowest classification efficiency. Despite lower level of repeatability, the PSO-FIS was able to produce structures with a $100 \%$ sensitivity. According to Table 1, differences between the employed soft computing classifiers are relatively small. The statistical significance analysis over obtained Dice index values has been performed using a Wilcoxon signed-rank test. In each cross validation scheme the SVM classifier outperforms all other classifiers at a confidence level of $p=0.05$. The other classifiers mostly do not differ significantly in various cross validation schemes except the $k=107$ case. A matrix of $p$-values obtained during verification of a null hypothesis for each pair of classifiers in a leave-one-out scheme $(k=107)$ is presented in Table 2, whilst corresponding Dice index box plot is shown in Fig. 7. The PSO-ANFIS tool features significant advantage here over all remaining classifiers (MLP, ANFIS, PSO-FIS). The classification accuracy metrics consistently exceeding $90 \%$ prove a high discriminant power of features describing cruciate ligaments.

The training time consumption reaches the largest level of ca. $30 \mathrm{~s}$ (performed on a workstation with CPU@3.40GHz, 16GB RAM, 64-bit Windows 7 OS and Matlab 7.14. - R2012a)

Table 1

Classification efficiency summary. Each classifier is evaluated in three modes of a $k$-fold cross validation $k=(3,10,107)$. Each cross-validation is repeated $N=20$ times with different database partitions. The left side of the table presents the averaged metrics, the right side - the best case metrics in terms of a Dice index

\begin{tabular}{|c|c|c|c|c|c|c|c|c|c|c|c|c|c|c|}
\hline & \multicolumn{7}{|c|}{ Average in $N$ runs } & \multicolumn{7}{|c|}{ Best case in $N$ runs } \\
\hline$k$ & $T P$ & $F P$ & $T N$ & $F N$ & $\operatorname{Sen}[\%]$ & Spec [\%] & $D[\%]$ & $T P$ & $F P$ & $T N$ & $F N$ & $\operatorname{Sen}[\%]$ & Spec [\%] & $D[\%]$ \\
\hline \multicolumn{15}{|c|}{ MLP } \\
\hline 3 & $58.8 \pm 4.6$ & $4.1 \pm 1.8$ & $40.9 \pm 1.8$ & $3.2 \pm 4.6$ & 94.8 & 90.9 & 94.1 & 60 & 1 & 44 & 2 & 96.8 & 97.8 & 97.6 \\
\hline 10 & $59.8 \pm 1.6$ & $3.4 \pm 1.5$ & $41.6 \pm 1.5$ & $2.2 \pm 1.6$ & 96.4 & 92.6 & 95.5 & 61 & 2 & 43 & 1 & 98.4 & 95.6 & 97.6 \\
\hline 107 & $60.0 \pm 0.9$ & $3.4 \pm 1.3$ & $41.6 \pm 1.3$ & $2.0 \pm 0.9$ & 96.8 & 92.3 & 95.7 & 61 & 2 & 43 & 1 & 98.4 & 95.6 & 97.6 \\
\hline \multicolumn{15}{|c|}{ SVM } \\
\hline 3 & $60.9 \pm 0.3$ & $3.2 \pm 0.4$ & $41.8 \pm 0.4$ & $1.1 \pm 0.3$ & 98.2 & 92.8 & 96.6 & 61 & 3 & 42 & 1 & 98.4 & 93.3 & 96.8 \\
\hline 10 & $61.0 \pm 0.0$ & $3.1 \pm 0.3$ & $41.9 \pm 0.3$ & $1.0 \pm 0.0$ & 98.4 & 93.1 & 96.7 & 61 & 3 & 42 & 1 & 98.4 & 93.3 & 96.8 \\
\hline 107 & $61.0 \pm 0.0$ & $3.0 \pm 0.0$ & $42.0 \pm 0.0$ & $1.0 \pm 0.0$ & 98.4 & 93.3 & 96.8 & 61 & 3 & 42 & 1 & 98.4 & 93.3 & 96.8 \\
\hline \multicolumn{15}{|c|}{ ANFIS } \\
\hline 3 & $60.2 \pm 1.4$ & $3.8 \pm 1.2$ & $41.2 \pm 1.2$ & $1.8 \pm 1.4$ & 97.2 & 91.7 & 95.6 & 61 & 2 & 43 & 1 & 98.4 & 95.6 & 97.6 \\
\hline 10 & $60.8 \pm 0.4$ & $3.6 \pm 0.7$ & $41.4 \pm 0.7$ & $1.2 \pm 0.4$ & 98.0 & 91.9 & 96.1 & 61 & 2 & 43 & 1 & 98.4 & 95.6 & 97.6 \\
\hline 107 & $60.8 \pm 0.4$ & $4.2 \pm 0.9$ & $40.8 \pm 0.9$ & $1.2 \pm 0.4$ & 98.1 & 90.6 & 95.7 & 61 & 2 & 43 & 1 & 98.4 & 95.6 & 97.6 \\
\hline \multicolumn{15}{|c|}{ PSO-FIS } \\
\hline 3 & $60.0 \pm 1.5$ & $3.8 \pm 0.9$ & $41.2 \pm 0.9$ & $2.0 \pm 1.5$ & 96.9 & 91.4 & 95.4 & 62 & 3 & 42 & 0 & 100.0 & 93.3 & 97.6 \\
\hline 10 & $60.1 \pm 1.3$ & $4.4 \pm 1.3$ & $40.6 \pm 1.3$ & $1.9 \pm 1.3$ & 96.9 & 90.1 & 95.0 & 62 & 4 & 41 & 0 & 100.0 & 91.1 & 96.9 \\
\hline 107 & $60.6 \pm 0.7$ & $4.3 \pm 1.3$ & $40.7 \pm 1.3$ & $1.4 \pm 0.7$ & 97.8 & 90.4 & 95.5 & 61 & 2 & 43 & 1 & 98.4 & 95.6 & 97.6 \\
\hline \multicolumn{15}{|c|}{ PSO-ANFIS } \\
\hline 3 & $60.0 \pm 1.1$ & $3.4 \pm 1.3$ & $41.6 \pm 1.3$ & $2.0 \pm 1.1$ & 96.8 & 92.4 & 95.7 & 61 & 1 & 44 & 1 & 98.4 & 97.8 & 98.4 \\
\hline 10 & $60.2 \pm 1.1$ & $3.6 \pm 0.9$ & $41.4 \pm 0.9$ & $1.8 \pm 1.1$ & 97.2 & 91.9 & 95.7 & 61 & 2 & 43 & 1 & 98.4 & 95.6 & 97.6 \\
\hline 107 & $60.7 \pm 0.6$ & $3.3 \pm 0.9$ & $41.7 \pm 0.9$ & $1.3 \pm 0.6$ & 97.9 & 92.7 & 96.3 & 61 & 2 & 43 & 1 & 98.4 & 95.6 & 97.6 \\
\hline
\end{tabular}


Table 2

A matrix of $p$-values obtained during the statistical significance analysis using Wilcoxon signed-rank test of all methods in terms of a Dice index (leave-one-out cross validation scheme). A table cell is highlighted with a gray background if a null hypothesis is rejected and two datasets of results differ significantly at a confidence level of $p=0.05$

\begin{tabular}{|c|c|c|c|c|l|}
\hline & MLP & SVM & ANFIS & $\begin{array}{l}\text { PSO- } \\
\text { FIS }\end{array}$ & $\begin{array}{l}\text { PSO- } \\
\text { ANFIS }\end{array}$ \\
\hline MLP & & 0.001 & 0.305 & 0.962 & 0.038 \\
\hline SVM & 0.001 & & 0.000 & 0.001 & 0.019 \\
\hline ANFIS & 0.305 & 0.000 & & 0.636 & 0.019 \\
\hline PSO-FIS & 0.962 & 0.001 & 0.636 & & 0.035 \\
\hline PSO-ANFIS & 0.038 & 0.019 & 0.019 & 0.035 & \\
\hline
\end{tabular}

in case of PSO-based classifiers specified as in Section 3. The particle fitness calculation procedure can be identified as the most time consuming part here since it requires a FIS/ANFIS evaluation per individual particle using all training vectors. Such an operation individually takes ca. $1 \mathrm{~ms}$, yet multiplied by the number of particles (here 50) consumes ca. $50 \mathrm{~ms}$ per iteration and by the number of iterations (here 500) - ca. $25 \mathrm{~s}$ per training.

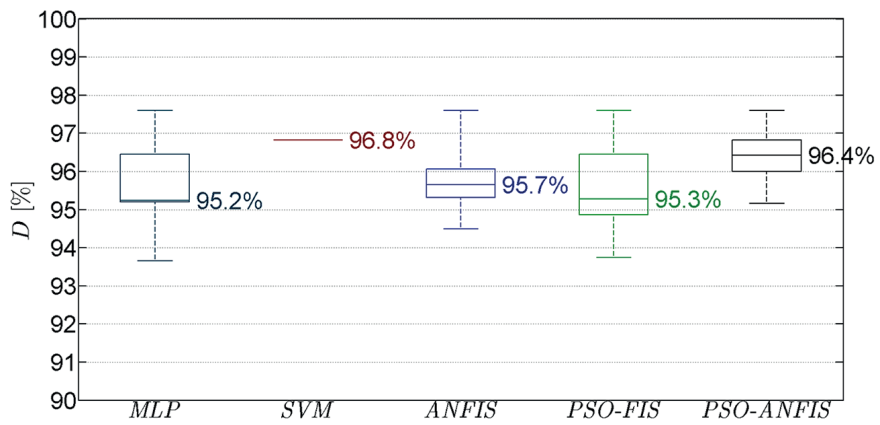

Fig. 7. Dice index box plot for a leave-one-out $(k=107)$ cross validation scheme. Each box covers a $25^{\text {th }}$ to $75^{\text {th }}$ percentile range with median value given and indicated by a central line and extreme values bordered by whiskers

\section{Conclusions}

The proposed workflow merges image analysis techniques to obtain a set of features describing the PCL structures and then employs classification stage for the computer aided PCL diagnosis. This approach can be helpful in clinical evaluation of PCL, especially in doubtful cases, where the visual assessment may be questionable. However, the effectiveness of the method in such challenging cases has to be investigated thoroughly. The proposed way provides a reliable and fast diagnosis of posterior cruciate ligaments, which plays very important role in the process of treatment and rehabilitation. The study conducted on selected features suitability for the PCL pathology assessment indicates relatively high level of sensitivity and specificity regardless of the employed classifier. The obtained results indicate that this concept may improve the diagnostic process of the PCL structures though its performance has to be confirmed using a study employing a larger set of cases of various difficulty level. The future work should focus on creating an intelligent, clinically applicable system dedicated to image analysis of the knee joint and advanced diagnostics of the cruciate ligaments with the methodology described in this paper standing for the first part of a full system.

Acknowledgements. This research was supported partially by the Polish Ministry of Science and Silesian University of Technology statutory financial support No. 211926/E-367/S/2016.

\section{REFERENCES}

[1] A. Bochenek and M. Reicher, "The human anatomy", PZWL, Warsaw, 1990, [in Polish].

[2] A. Pasierbinski and A. Jarzabek, "Biobiomechanics of the cruciate ligament", Acta Clinica 4(1), 284-293 (2001), [in Polish].

[3] J. Voos, C. Mauro, T. Wente, R. Warren, and T. Wickiewicz, "Posterior cruciate ligament. Anatomy, biomechanics and outcomes", Am. J. Sports. Med. 40(1), 222-231 (2012).

[4] A. Dziak, "Injuries of the cruciate ligaments of the knee joint", Acta Clinica 4(1), 271-274 (2001), [in Polish].

[5] P. Zarychta, "Features extraction in anterior and posterior cruciate ligaments analysis", Comput. Med. Imag. Grap. 46, Part 2, 108-120 (2015).

[6] M. Aiello, "MRI for posterior cruciate ligament injuries", available online: http://emedicine.medscape.com/article/400845- overview [accessed 2016-06-06].

[7] A. Alcala-Galiano, M. Baeva, M. Ismael, and M. Argueso, "Imaging of posterior cruciate ligament (PCL) reconstruction: normal postsurgical appearance and complications", Skeletal Radiol. 43(12), 1659-1668 (2014).

[8] F.R. Noyes, and S.D. Barber-Westin, Noyes'knee disorders: surgery, rehabilitation, clinical outcomes, 2nd ed., Elsevier, 2017.

[9] P. Zarychta, "Posterior cruciate ligament - 3D visualization", in Conference on Computer Recognition Systems, Advances in Intelligent and Soft Computing 45, 695-702 (2007).

[10] R. Wright et al., "Outcome of revision anterior cruciate ligament reconstruction: a systematic review", J. Bone Joint Surg. Am. 94(6), 531-536 (2012).

[11] B. Crespo, E. James, L. Metsavaht, and R. LaPrade, "Injuries to posterolateral corner of the knee: a comprehensive review from anatomy to surgical treatment", Revista Brasileira de Ortopedia (English Edition) 50(4), 363-370 (2015).

[12] D. Hensler, C. Van Eck, F. Fu, and J. Irrgang, "Anatomic anterior cruciate ligament reconstruction utilizing the doublebundle technique", J. Orthop. Sport. Phys. 42(3), 184-195 (2012).

[13] S. Czuppon, B. Racette, S. Klein, and M. Harris-Hayes, "Variables associated with return to sport following anterior cruciate ligament reconstruction: a systematic review", Brit. J. Sport. Med. 48, 356-364 (2014).

[14] L. Kruse, B. Gray, and L. Wright, "Rehabilitation after anterior cruciate ligament reconstruction”, J. Bone Joint Surg. Am. 94(19), 1737-1748 (2012).

[15] P. Glasgow, "Simplicity: the ultimate sophistication", Brit. J. Sport. Med. 48, 345 (2014). 
[16] J. Cavanaugh, A. Saldivar, and R. Marx, "Postoperative rehabilitation after posterior cruciate ligament reconstruction and combined posterior cruciate ligament reconstruction-posterior lateral corner surgery", Oper. Techn. Sport. Med. 23(4), 372-384 (2015).

[17] P. Zarychta, "Cruciate ligaments of the knee joint in the computer analysis", in Information Technologies in Biomedicine, Advances in Intelligent Systems and Computing 283, 71-80 (2014).

[18] P. Zarychta, "ACL and PCL of the knee joint in the computer diagnostics", in 21st International Conference Mixed Design of Integrated Circuits and Systems MIXDES2014, 489-492 (2014).

[19] A. Zarychta-Bargiela, P. Zarychta, "The importance of the features of the posteriori cruciate ligament in diagnosis", in Information Technologies in Medicine, Advances in Intelligent Systems and Computing 471, 165-177 (2016).

[20] W. Wieclawek and E. Pietka, "Watershed based intelligent scissors", Comput. Med. Imag. Grap. 43, 122-129 (2015).

[21] P. Zarychta and A. Zarychta-Bargiela, "Anterior and posterior cruciate ligament-extraction and 3D visualization", in Information Technologies in Biomedicine, Advances in Intelligent and Soft Computing 69, 115-122 (2010).

[22] M. Young, The technical writer's handbook, Mill Valley, Toronto, 1989.

[23] R.A. Fisher, "The use of multiple measurements in taxonomic problems", Annals of Eugenics 7(2), 179-188 (1936).

[24] S. Haykin, Neural networks: a comprehensive foundation, 2nd ed., Prentice Hall PTR, USA, 1998.

[25] C. Cortes and V. Vapnik, "Support-vector networks", Mach. Learn. 20(3), 273-297 (1995).

[26] J. Czajkowska, M. Rudzki, and Z. Czajkowski, "A new fuzzy support vectors machine for biomedical data classification", in 30th Annual International Conference of the IEEE Engineering in Medicine and Biology Society, 4676-4679 (2008).

[27] J.S.R. Jang, "ANFIS: adaptive-network-based fuzzy inference system”, IEEE T. Syst. Man Cyb. 23(3), 665-685 (1993).

[28] T. Takagi and M. Sugeno, "Fuzzy identification of systems and its applications to modeling and control", IEEE T. Syst. Man Cyb. 15, 116-132 (1985).
[29] J. Kawa and E. Pietka, "Image clustering with median and myriad spatial constraint enhanced FCM", in Conference on Computer Recognition Systems, Advances in Soft Computing, 211-218 (2005).

[30] J. Kennedy, R.C. Eberhart, "Particle swarm optimization", in Proc. IEEE International Conference on Neural Networks, 19421948 (1995).

[31] M.M. Millonas, "Swarms, phase transitions, and collective intelligence", in Artificial Life III, Addison-Wesley (1994).

[32] H.J. Escalante, et al., "Acute leukemia classification by ensemble particle swarm model selection", Artif. Intell. Med. 55, 163-175 (2012).

[33] C. Guarnizo, A. A. Orozco, M. A. Alvarez, "Optimal sampling frequency in wavelet-based signal feature extraction using particle swarm optimization", in 35th Annual International Conference of the IEEE Engineering in Medicine and Biology Society, 993-996 (2013).

[34] S. Alam, G. Dobbie, Y. S. Koh, P. Riddle, S. U. Rehman, "Research on particle swarm optimization based clustering: a systematic review of literature and techniques", Swarm and Evolutionary Computation 17, 1-13 (2014).

[35] I. Zyout, J. Czajkowska, M. Grzegorzek, "Multi-scale textural feature extraction and particle swarm optimization based model selection for false positive reduction in mammography", Comput. Med. Imag. Grap. 46, Part 2, 95-107 (2015).

[36] M. Galinska, P. Badura, "Swarm intelligence approach to 3D medical image segmentation", in Information Technologies in Medicine, Advances in Intelligent Systems and Computing 471, 15-24 (2016).

[37] P. Badura, E. Pietka, "3D fuzzy liver tumor segmentation", in Information Technologies in Biomedicine, Lecture Notes in Bioinformatics 7339, 47-57 (2012).

[38] M. V. Oliveira and R. Schirru, "Applying particle swarm optimization algorithm for tuning a neuro-fuzzy inference system for sensor monitoring”, Prog. Nucl. Energ. 51(1), 177-183 (2009).

[39] S. Arlot, A. Celisse, "A survey of cross-validation procedures for model selection", Statistics Surveys 4, 40-79 (2010).

[40] J. Kawa, J. Juszczyk, B. Pycinski, P. Badura, and E. Pietka, "Radiological atlas for patient specific model generation", in Information Technologies in Biomedicine, Advances in Intelligent Systems and Computing 284 (4), 69-82 (2014). 